\title{
Currículo de Odontopediatria nos cursos brasileiros de graduação em Odontologia
}

\author{
Vanessa Silva da Costa*; Marcia Turolla Wanderley**; Ana Estela Haddad**; Karla Mayra

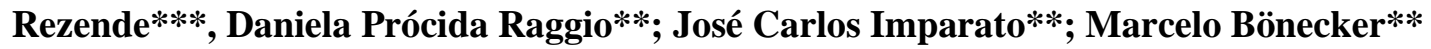

* Especialista em Odontopediatria, Faculdade de Odontologia da Universidade de São Paulo

** Professor(a), Disciplina de Odontopediatria, Faculdade de Odontologia da Universidade de São Paulo

*** Pós-doutoranda em Odontopediatria, Faculdade de Odontologia da Universidade de São Paulo

Recebido em 22/10/2019. Aprovado em $21 / 06 / 2020$.

\begin{abstract}
RESUMO
O objetivo deste estudo foi coletar informações relativas aos conteúdos de Odontopediatria desenvolvidos nos cursos de graduação em Odontologia brasileiros. Para a coleta dos dados foi utilizado um questionário eletrônico, enviado a cada curso com a solicitação de que fosse respondido por um professor da área. Em janeiro de 2017, segundo registros no sistema e-MEC, 372 instituições ofereciam o curso de Odontologia no Brasil. Dessas, 76 responderam que o curso era novo e que esses conteúdos ainda não tinham sido ofertados. Assim, a amostra total foi inicialmente reduzida para 296 instituições, entre as quais 139 responderam o questionário (taxa de resposta=47\%). A disciplina de Odontopediatria foi geralmente oferecida em dois semestres $(42,4 \%)$, com carga horária média de 84,5 horas para atividades teóricas e 34,2 horas para a parte laboratorial. Observou-se que 35,3\% dos cursos não possuíam atividades laboratoriais. Sobre o atendimento clínico, $38,1 \%$ não atendiam a crianças de 0 a 3 anos e a média de horas de atendimento clínico a crianças maiores de 3 anos foi de 119,3 horas. Os elementos e dimensões aqui tratados devem nortear o aprofundamento das investigações sobre a qualidade da formação no que diz respeito ao perfil generalista por um lado, e por outro, ao currículo da disciplina de Odontopediatria.

Descritores: Avaliação Educacional. Educação Superior. Odontopediatria. Odontologia. Currículo.
\end{abstract}




\section{INTRODUÇÃO}

O primeiro curso de Odontologia do Brasil surgiu por meio do Decreto n. 9.311, de 25 de outubro de 1884, que regulamentou a criação de cursos de Odontologia, Farmácia e Obstetrícia anexos às Faculdades de Medicina. Na época, as duas únicas Faculdades de Medicina do país estavam no Rio de Janeiro e em Salvador. Nesse decreto foram descritas as matérias que deveriam obrigatoriamente ser ministradas no curso de Odontologia, entre elas ainda não havia a disciplina de Odontopediatria ${ }^{1,2}$.

Com a criação do curso de Odontologia independente das Faculdades de Medicina, o currículo foi sendo ampliado e, com a fundação da Universidade de São Paulo, em janeiro de 1934, e a reformulação do currículo, foi criada a $12^{\mathrm{a}}$ cadeira de Clínica de Ortodontia e Odontopediatria ${ }^{4}$. Em 2002, com o advento das Diretrizes Curriculares Nacionais do Curso de Graduação de Odontologia (Resolução CNE/CES n. 3/2002), foram estabelecidas normas básicas para o curso, entre essas regras estavam os conteúdos essenciais que deveriam ser ministrados, sendo eles ${ }^{3}$ : I - Ciências Biológicas e da Saúde; II - Ciências Humanas e Sociais e III - Ciências Odontológicas (Propedêutica Clínica, Clínica Odontológica e Odontologia Pediátrica).

As diretrizes tiveram como objetivo estabelecer as competências mínimas do cirurgiãodentista ao final da sua graduação, não especificando qual seria o objetivo de cada disciplina do curso; o que difere do manual publicado em 2009 pela Academia Europeia de Odontopediatria (EAPD $)^{5}$, que especifica os objetivos da disciplina na graduação, além das habilidades e aptidões que cada estudante deve adquirir durante sua formação. No Brasil não existe algo similar.

Nesse sentido, considerando as competências a serem desenvolvidas durante o curso de graduação com relação aos conteúdos de
Odontopediatria, o objetivo deste estudo foi coletar e comparar as informações sobre o currículo da área nas instituições que oferecem o curso de Odontologia no Brasil.

\section{METODOLOGIA}

Foi realizado um estudo transversal descritivo aprovado pelo Comitê de Ética em Pesquisa da Faculdade de Odontologia da Universidade de São Paulo (FOUSP) (CAAE 83759418.7.0000.0075 e parecer de aprovação $n^{\circ}$. 2.523.026). Inicialmente foi elaborado um questionário eletrônico utilizando a plataforma Google Forms. Esse questionário era composto por 30 perguntas, sendo 23 discursivas e 7 de múltipla escolha. Os temas principais das perguntas foram assim agrupados: informações gerais sobre o curso de Odontologia; conteúdo e formato das aulas teóricas em Odontopediatria; conteúdo e formato das aulas práticas em laboratório de Odontopediatria; conteúdo e formato do ensino a distância em Odontopediatria; conteúdo e formato das aulas práticas em clínica infantil para crianças de até 3 anos de idade; e conteúdo e formato das aulas práticas em clínica infantil para pacientes maiores de 3 anos de idade.

As respostas foram obrigatórias para todas as perguntas. $O$ sigilo foi garantido pela não identificação prévia ao envio das respostas.

Esse questionário foi testado em estudo piloto, com estudantes do curso de pós-graduação em Odontopediatria da FOUSP, para aferir a facilidade de preenchimento e entendimento das questões elaboradas.

Os dados dos cursos foram coletados do sistema e-MEC 6 . Em 2017, quando iniciado o estudo, havia 372 instituições que ofereciam o curso de Odontologia. Algumas ofereciam mais de um curso, um diurno e outro noturno, perfazendo o total de 381 cursos de graduação em Odontologia oferecidos no Brasil.

Para obter o contato de um professor de 
Odontopediatria de cada curso, obteve-se o apoio da Associação Brasileira de Odontopediatria (ABOPED), que solicitou aos presidentes das regionais de cada estado brasileiro que tentassem localizar o endereço eletrônico de pelo menos um dos professores de Odontopediatria de cada curso de Odontologia de seu respectivo estado.

Além disso, a pesquisadora principal realizou contato telefônico com a Secretaria Geral de várias instituições para obter informações de contato de professores de Odontopediatria. Do total de 372 instituições, 76 responderam que o curso de Odontologia era novo e ainda não tinham ofertado a disciplina de Odontopediatria, dessa maneira, a amostra total foi inicialmente reduzida para 296 cursos. Não foi possível obter o contato de um professor de Odontopediatria em 115 cursos de Odontologia e em 42 os professores foram convidados, mas não responderam. Assim, a amostra final foi composta por 139 questionários (figura 1).

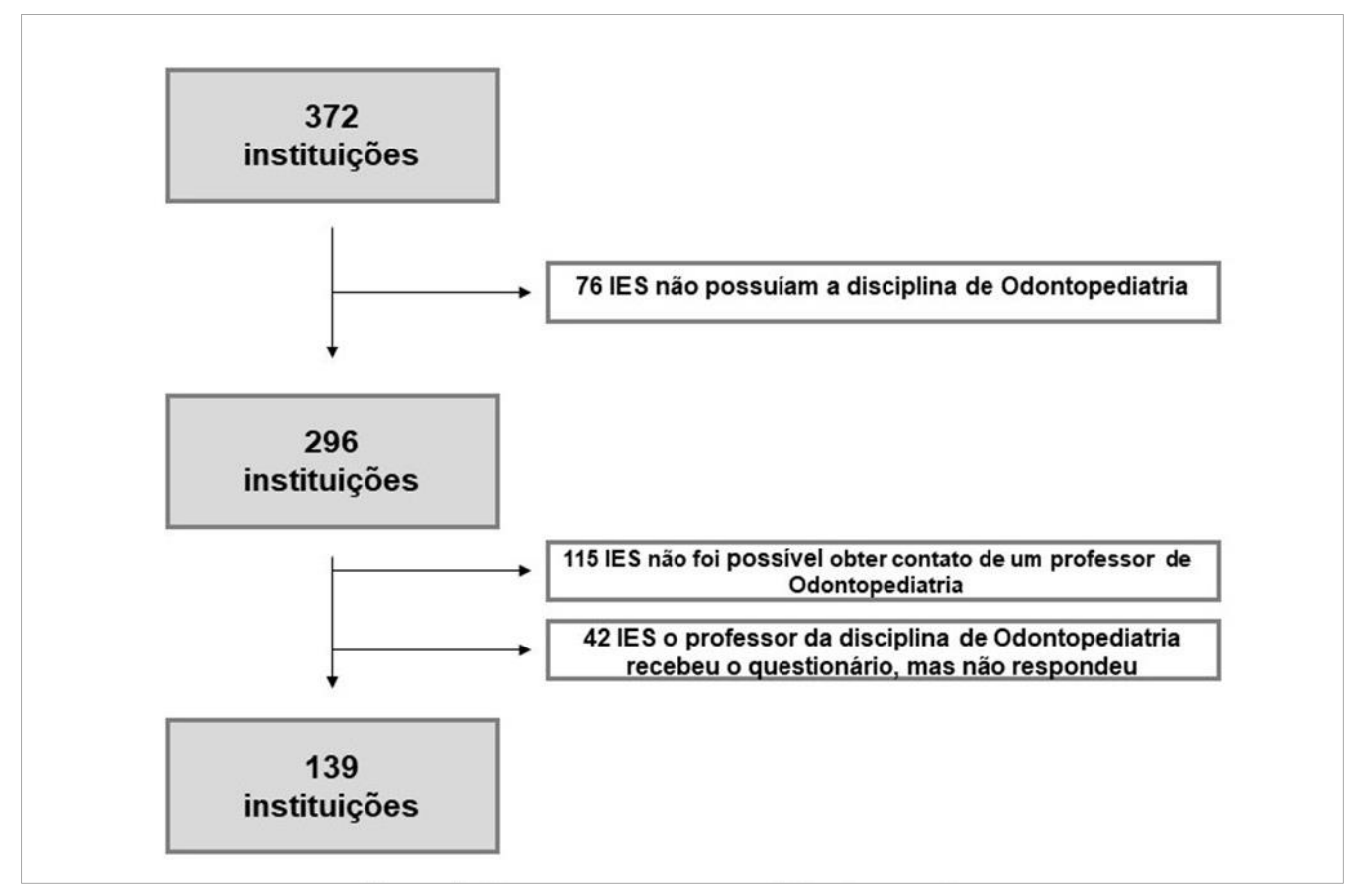

Figura 1. Fluxograma com a descrição da amostra

Os participantes receberam um e-mail que os convidava a participar da pesquisa, informava os objetivos e a metodologia utilizada, assim como os links para acessar o Termo de Consentimento Livre e Esclarecido e o questionário eletrônico.

As respostas foram tabuladas no software Excel (Redmond, WA, EUA), e submetidas a análises descritivas. Quando houve respostas de mais de um professor da mesma instituição, foi considerada apenas a primeira.

\section{RESULTADOS}

Dos 296 cursos potencialmente elegíveis para o estudo, foram respondidos 139 questionários, sendo a taxa de resposta global de $47 \%$.

Do total de instituições que oferecem o curso de Odontologia, 317 são privadas, o que corresponde a $85,2 \%$ do total, contra 55 públicas 
(14,8\%). Entre as instituições públicas então uma ainda não ofertava a disciplina. A figura 2 existentes, $45(81,8 \%)$ participaram do estudo e apresenta a distribuição das instituições por estado.

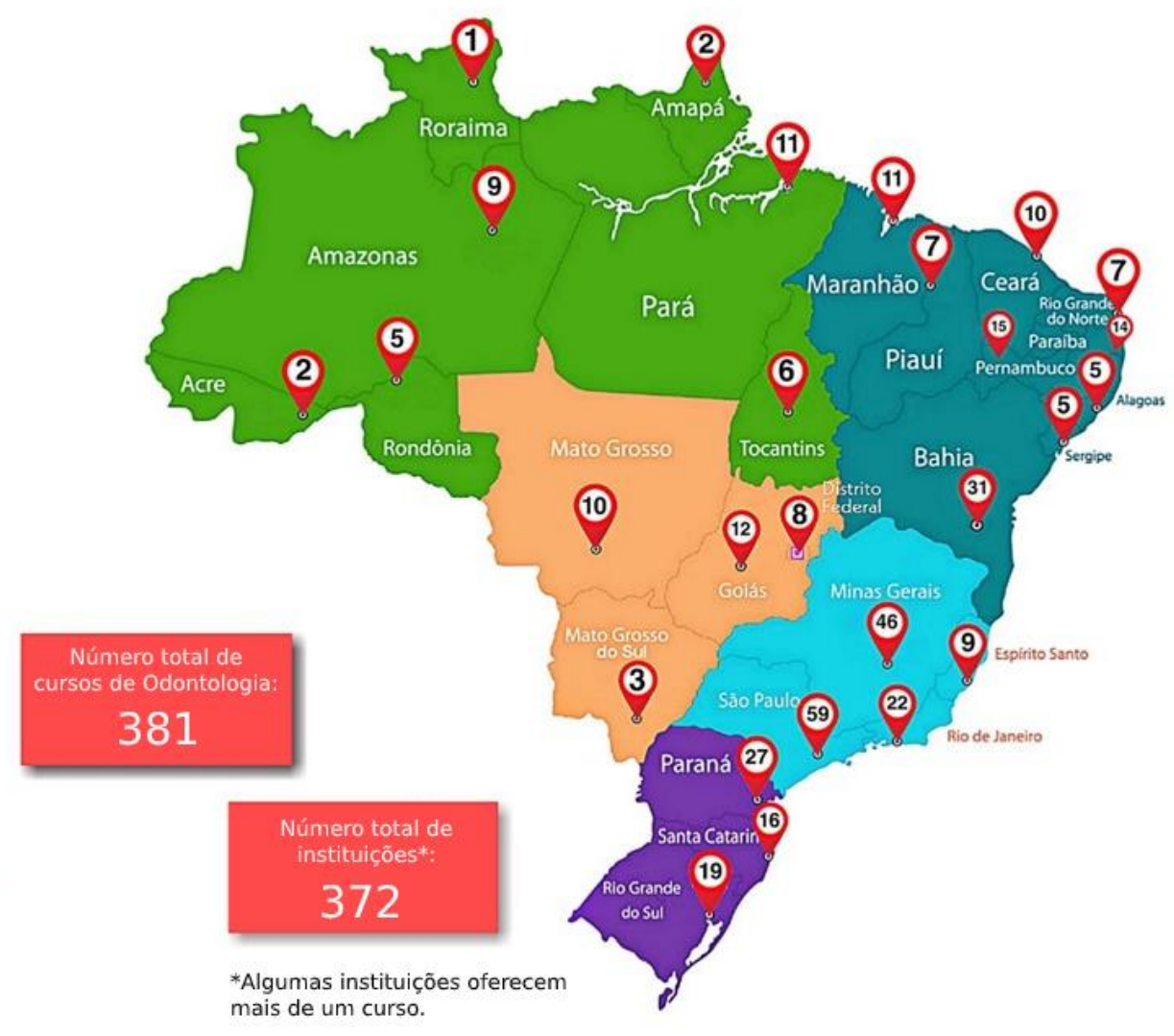

Figura 2. Distribuição dos cursos de Odontologia nos estados brasileiros.

Das 139 instituições que responderam ao questionário, $100(71,9 \%)$ ofereciam apenas um curso, $32(23 \%)$ ofereciam dois e sete $(5 \%)$ ofereciam três, totalizando 185 cursos, sendo 104 $(56,2 \%)$ em período integral, $32(17,3 \%)$ no período matutino, $21(11,4 \%)$ no período vespertino e $28(15,1 \%)$ no período noturno.

Em 13 cursos $(9,4 \%)$ a disciplina ocorre em um único semestre, em $59(42,4 \%)$ em dois, em 45 $(32,4 \%)$ em três, em $16(11,5 \%)$ em quatro e em 6 $(4,3 \%)$ em cinco ou mais semestres, sendo ministrada com mais frequência entre o $6^{\circ}$ e $9^{\circ}$ semestres. Em 45 (32,4\%) cursos a disciplina foi ministrada em conjunto com a Ortodontia.

A média de carga horária de aulas teóricas foi de 84,5 horas, variando entre 12 e 320 horas. Em relação às atividades laboratoriais, a média foi de 34,2 horas, variando entre 0 (49 cursos $-35,5 \%$ ) e 384 horas, mas 49 cursos $(35,3 \%)$ não possuem atividades laboratoriais. As atividades laboratoriais mais frequentes foram tratamentos endodônticos, confecção de aparelhos ortodônticos móveis utilização de materiais odontológicos e técnicas diretas e indiretas e diagnóstico de cárie.

Quanto às atividades clínicas, $53(38,1 \%)$ responderam que não atendem crianças de 0-3 anos na clínica da graduação, $22(15,8 \%)$ destinam de uma a quatro horas de atendimento durante o curso para essa faixa etária, 7 (5\%) de quatro a oito horas, $6(4,3 \%)$ de 8 a 12 horas e $51(36,7 \%)$ mais de 12 
horas (tabela 1). Quanto ao atendimento de crianças maiores de 3 anos, a média de carga horária foi de 119,3 horas, variando de 0 a 600 horas.

Em média, 2,7 horas são utilizadas em ensino a distância (EaD), contudo 108 (77,7\%) cursos não usavam essa forma de ensino complementar. Os cursos que utilizavam o EAD realizaram como atividades a oferta de textos e exercícios complementares às aulas teóricas.

A variação de horas aula para cada tema pode ser observada na tabela 2. Para o tema prevenção, diagnóstico e tratamento de desgaste dentário erosivo, 48 cursos não abordaram esse tema, enquanto 24 ministraram mais de 2 horas/aula sobre o assunto. A abordagem de mínima intervenção para todos os conteúdos foi relatada por 133 cursos $(95,7 \%)$.

$\mathrm{Na}$ pergunta sobre a existência ou não de algum tema do currículo de Odontopediatria que não tivesse sido abordado e que achavam importante destacar, as respostas mais frequentes foram Periodontia na infância e na adolescência, Disfunção temporomandibular e bruxismo, Patologia, Anatomia dos dentes decíduos, Odontohebiatria, Reabilitação protética, Abordagem e motivação do paciente infantil e da sua família, Maus-tratos, Gestante e pré-natal odontológico, Biossegurança e Emergências médicas.

\section{DISCUSSÃO}

Os dados coletados nesta pesquisa permitem vislumbrar como a disciplina de Odontopediatria vem sendo ministrada no Brasil. Notou-se discrepâncias quantitativas e qualitativas entre os currículos desenvolvidos nos diversos cursos de graduação.

No aspecto quantitativo, observa-se a expressiva diferença da carga horária destinada ao ensino de Odontopediatria. Existem instituições que oferecem a disciplina em apenas um semestre durante todo o curso de graduação $(9,3 \%)$, enquanto outras oferecem o curso em cinco ou mais semestres $(4,3 \%)$, mostrando uma grande variedade de carga horária. A maioria $(42,4 \%)$, no entanto, oferece o curso em dois semestres. Ao analisar, separadamente, a carga horária de cada curso, pode-se notar que as diferenças ficam mais evidentes. Em relação às aulas teóricas, observa-se uma discrepância não só quantitativa, mas também qualitativa.

Em relação à carga horária teórica, embora a média tenha sido de 84,5 horas, existem instituições que oferecem apenas 12 horas de aula, enquanto outras oferecem 320. Essa diferença se reflete também no conteúdo, pois, ao se observar a quantidade de horas designadas para cada tema nota-se que alguns deles, como desgaste dentário erosivo, hipomineralização molar incisivo e pacientes especiais não são abordados na disciplina em alguns dos cursos. Se considerarmos que o objetivo dos cursos é formar cirurgiões-dentistas capazes de promover a saúde bucal da população em geral $^{3}$, inclusive da população infantil, a ausência desses conteúdos pode comprometer a apreensão da filosofia de mínima intervenção e a visão integral da saúde bucal das crianças.

Nas atividades laboratoriais também se percebeu que há diferenças entre os cursos, pois em $49(35,3 \%)$ deles não havia atividades laboratoriais. A literatura ressalta a importância do treinamento pré-clínico para a formação dos cirurgiões-dentistas $^{7,8}$, que contribui para a sedimentação e integração entre o conteúdo teórico e o processamento necessário para que o estudante adquira habilidades para sua aplicação na prática clínica.

Sobre as atividades clínicas, o tema que teve a maior discrepância foi a clínica de bebês. $O$ atendimento de crianças menores que 3 anos de idade não foi realizado em $38,1 \%$ das instituições e, segundo as justificativas, os principais motivos seriam não haver professores em número suficiente para auxiliar os estudantes nesse tipo de atendimento; 
insuficiente carga horária; ou esse atendimento era realizado em estágio ou cursos de extensão.

Tabela 1. Distribuição da carga horária das disciplinas de Odontopediatria

\begin{tabular}{|c|c|c|c|c|c|c|c|c|c|c|c|}
\hline \multirow[t]{2}{*}{ Variáveis } & \multicolumn{2}{|c|}{0 horas } & \multicolumn{2}{|c|}{$1-4$ horas } & \multicolumn{2}{|c|}{$4-8$ horas } & \multicolumn{2}{|c|}{$8-12$ horas } & \multicolumn{2}{|c|}{$>12$ horas } & \multirow[t]{2}{*}{ Total } \\
\hline & $\mathbf{n}$ & $\%$ & $\mathbf{n}$ & $\%$ & $\mathbf{n}$ & $\%$ & $\mathbf{n}$ & $\%$ & $\mathbf{n}$ & $\%$ & \\
\hline $\begin{array}{l}\text { Atividades } \\
\text { laboratoriais }\end{array}$ & 53 & $38,1 \%$ & 5 & $3,6 \%$ & 10 & $7,2 \%$ & 12 & $8,6 \%$ & 59 & $42,4 \%$ & 139 \\
\hline $\begin{array}{c}\text { Clínica de bebês } \\
\text { anos) }\end{array}$ & 53 & $38,1 \%$ & 22 & $15,8 \%$ & 7 & $5 \%$ & 6 & $4,3 \%$ & 51 & $36,7 \%$ & 139 \\
\hline $\begin{array}{c}\text { Clínica infantil } \\
\text { (maiores de } 3 \text { anos) }\end{array}$ & 1 & $0,7 \%$ & 0 & $0,0 \%$ & 2 & $1,4 \%$ & 5 & $3,6 \%$ & 131 & $94,2 \%$ & 139 \\
\hline
\end{tabular}

Tabela 2. Distribuição de carga horária de temas abordados nas aulas teóricas

\begin{tabular}{|c|c|c|c|c|c|c|c|c|c|c|}
\hline \multirow{2}{*}{ Tema } & \multicolumn{2}{|c|}{ Nenhuma } & \multicolumn{2}{|c|}{1 hora } & \multicolumn{2}{|c|}{2 horas } & \multicolumn{2}{|c|}{$>2$ horas } & \multirow{2}{*}{ Média } & \multirow[b]{2}{*}{ Total } \\
\hline & $\mathbf{n}$ & $\%$ & $\mathbf{n}$ & $\%$ & $\mathbf{N}$ & $\%$ & $\mathbf{n}$ & $\%$ & & \\
\hline Prevenção de cárie dental & 1 & $0,7 \%$ & 3 & $2,2 \%$ & 23 & $16,5 \%$ & 112 & $80,6 \%$ & 6,9 & 139 \\
\hline Diagnóstico de cárie dental & 3 & $2,2 \%$ & 8 & $5,8 \%$ & 36 & $25,9 \%$ & 92 & $66,2 \%$ & 3,9 & 139 \\
\hline Tratamento de lesões de cárie & 0 & - & 1 & $0,7 \%$ & 24 & $17,3 \%$ & 114 & $82 \%$ & 5,4 & 139 \\
\hline $\begin{array}{l}\text { Prevenção, tratamento e diagnóstico } \\
\text { de traumatismo dentário }\end{array}$ & 0 & - & 3 & $2,2 \%$ & 22 & $15,8 \%$ & 114 & $82 \%$ & 4,8 & 139 \\
\hline $\begin{array}{l}\text { Prevenção, tratamento e diagnóstico } \\
\text { de mal oclusão }\end{array}$ & 24 & $17,3 \%$ & 7 & $5 \%$ & 22 & $15,8 \%$ & 86 & $61,9 \%$ & 6,3 & 139 \\
\hline $\begin{array}{l}\text { Prevenção, tratamento e diagnóstico } \\
\text { de desgaste dentário erosivo }\end{array}$ & 48 & $34,5 \%$ & 24 & $17,3 \%$ & 43 & $30,9 \%$ & 24 & $17,3 \%$ & 1,5 & 139 \\
\hline $\begin{array}{l}\text { Prevenção, tratamento e diagnóstico } \\
\text { de HMI }\end{array}$ & 27 & $19,4 \%$ & 34 & $24,5 \%$ & 50 & $36 \%$ & 28 & $20,1 \%$ & 1,8 & 139 \\
\hline $\begin{array}{l}\text { Controle não farmacológico do } \\
\text { paciente }\end{array}$ & 3 & $2,2 \%$ & 5 & $3,6 \%$ & 45 & $32,4 \%$ & 86 & $61,9 \%$ & 3,8 & 139 \\
\hline Controle farmacológico do paciente & 37 & $26,6 \%$ & 24 & $17,3 \%$ & 48 & $34,5 \%$ & 30 & $21,6 \%$ & 1,9 & 139 \\
\hline Tratamento endodôntico & 0 & - & 2 & $1,4 \%$ & 17 & $12,2 \%$ & 120 & $86,3 \%$ & 5,3 & 139 \\
\hline $\begin{array}{l}\text { Tratamento cirúrgico de baixa } \\
\text { complexidade }\end{array}$ & 2 & $1,4 \%$ & 15 & $10,8 \%$ & 60 & $43,2 \%$ & 62 & $44,6 \%$ & 2,9 & 139 \\
\hline $\begin{array}{l}\text { Tratamento cirúrgico de alta } \\
\text { complexidade }\end{array}$ & 20 & $14,4 \%$ & 14 & $10,1 \%$ & 62 & $44,6 \%$ & 43 & $30,9 \%$ & 2,3 & 139 \\
\hline Pacientes com necessidades especiais & 70 & $50,4 \%$ & 10 & $7,2 \%$ & 28 & $20,1 \%$ & 31 & $22,3 \%$ & 2,5 & 139 \\
\hline Odontologia para bebês & 9 & $6,5 \%$ & 6 & $4,3 \%$ & 61 & $43,9 \%$ & 63 & $45,3 \%$ & 3,9 & 139 \\
\hline Prescrição medicamentosa & 7 & $5 \%$ & 15 & $10,8 \%$ & 55 & $39,6 \%$ & 62 & $44,6 \%$ & 3 & 139 \\
\hline $\begin{array}{l}\text { Técnicas radiográficas e } \\
\text { interpretação de imagens }\end{array}$ & 2 & $1,4 \%$ & 11 & $7,9 \%$ & 60 & $43,2 \%$ & 66 & $47,5 \%$ & 3,1 & 139 \\
\hline Anestesiologia & 0 & - & 12 & $8,6 \%$ & 68 & $48,9 \%$ & 59 & $42,4 \%$ & 3 & 139 \\
\hline EaD & 108 & $77,7 \%$ & 1 & $0,7 \%$ & 2 & $1,4 \%$ & 28 & $20,1 \%$ & 2,7 & 139 \\
\hline
\end{tabular}


$\mathrm{O}$ atendimento de crianças com menos de 3 anos de idade é importante na graduação, visto que o cirurgião-dentista generalista deve ser capacitado para reconhecer aspectos de normalidade e de doença, ter conhecimento sobre o crescimento e o desenvolvimento, uma vez que, normalmente, os pais desses pacientes de pouca idade procuram primeiro seus próprios dentistas para receber as primeiras orientações sobre a saúde bucal dos seus filhos $^{3}$ no âmbito de serviços particulares. É importante destacar também que no serviço público, o cirurgião-dentista generalista fica responsável pela prevenção e promoção de saúde bucal de todos os membros da família atendida pelo seu serviço de saúde.

Segundo a Academia Americana de Odontopediatria ${ }^{9,10}$, a primeira consulta do bebê deve ser feita na época da erupção do primeiro dente, antes do primeiro ano de vida. O acompanhamento da erupção dos dentes, da oclusão, medidas preventivas e tratamento precoce de lesões de cárie melhora a saúde bucal e geral do bebê, reforçando a importância de o clínico geral ter alguma familiaridade com esse público.

A expansão quantitativa de cursos de Odontologia oferecidos no país ${ }^{2,11}$ e os resultados encontrados neste estudo retratando grandes discrepâncias, tanto nos aspectos relativos à carga horária total como na distribuição desta carga horária entre as diferentes atividades e mesmo nos conteúdos que compõem a disciplina de Odontopediatria, nos fazem despertar para questões sobre como podemos aferir se os diferentes cursos têm alcançado os objetivos de formação com qualidade dos cirurgiões-dentistas no Brasil, no que diz respeito a atenção à saúde bucal de bebês, crianças e adolescentes.

As DCN não preconizam a padronização de conteúdos herméticos e prescritivos, mas sim abordagens mais contemporâneas e que tomam em consideração uma série de condições, como por exemplo as características de cada população onde o curso e a instituição de ensino se inserem, o perfil socioepidemiológico e as necessidades de saúde da população, entre outros.

O Sistema Nacional de Avaliação da Educação Superior (SINAES) prevê a diversidade nos Planos de Desenvolvimento Institucional das instituições, como também nos projetos político pedagógicos dos cursos.

A Associação Brasileira de Odontopediatria vem editando o Manual de Referência para Procedimentos Clínicos em Odontopediatria ${ }^{12}$, num esforço de especialistas de todo o país, tratando dos temas que compõem a especialidade, sempre atualizados com as melhores evidências científicas. A especialidade da Odontopediatria brasileira tem uma história relativamente recente e, no entanto, avançou muito no corpo de conhecimentos, experiência e filosofia de atenção integral à saúde bucal de bebês, crianças e adolescentes, tanto no serviço público como no privado $^{13}$, alcançando projeção e reconhecimento internacional.

O diagnóstico inicial trazido por esta pesquisa nos leva à percepção sobre a necessidade de novos estudos nos quais se possa aferir, com abordagens complementares, seja da perspectiva docente, de coordenadores de curso, mas também dos estudantes, a qualidade do ensino da Odontopediatria nos cursos de graduação em Odontologia.

\section{CONCLUSÕES}

A comparação entre diversos cursos de graduação em Odontologia demonstrou a grande disparidade existente nos currículos da disciplina de Odontopediatria. A discrepância é bastante ampla tanto na carga horária total, como na sua distribuição entre atividades teóricas, laboratoriais e clínicas, como também na ausência de determinados conteúdos considerados relevantes, em alguns cursos. A utilização complementar da educação a distância é ainda inexistente na maioria 
dos cursos e em mais de um terço dos cursos os estudantes não são capacitados para o atendimento de bebês. Os elementos e dimensões aqui tratados devem nortear o aprofundamento das investigações sobre a qualidade da formação no que diz respeito ao perfil generalista por um lado, e por outro ao currículo da disciplina de Odontopediatria.

\section{AGRADECIMENTOS}

Os autores agradecem aos professores de Odontopediatria das instituições que participaram respondendo ao questionário, ao Lucas Brum por ter auxiliado com a ilustração.

\section{ABSTRACT \\ Paediatric Dentistry curriculum in under- graduate Dental courses in Brazil}

This study aimed to collect information regarding the contents of paediatric dentistry developed in Brazilian undergraduate dental courses. For data collection, an electronic questionnaire was sent to each course and answered by the teacher of the discipline. In January 2017, according to the E-MEC website, 372 institutions offered dental courses in Brazil. Of these, 76 answered that the course was new and the contents had not yet been actually offered. Thus, the total sample was initially reduced to 296 institutions, among which 139 answered the questionnaire (response rate $=$ $47 \%$ ). The discipline of paediatric dentistry was generally offered in two semesters $(42.4 \%)$, with an average workload of 84.5 hours for theoretical activities, 34.2 hours for laboratory practices. It was observed that $35.3 \%$ of the courses did not have laboratory activities. With regard to clinical activities, $38.1 \%$ did not provide care to children aged 0 to 3 years old and the average hours of clinical care for children older than 3 years old was 119.3 hours. The elements and dimensions discussed here should guide further investigation into the quality of training of the general dentist on the one hand, and on the other, into the curriculum of the discipline of paediatric dentistry.

Descriptors: Educational Measurement.
Education, Higher. Paediatric Dentistry. Dentistry. Curriculum.

\section{REFERÊNCIAS}

1. Brasil. Decreto $n^{\circ} 9311$ de 25 de outubro de 1884. Coleção das Leis do Império do Brasil de 1884. Parte I. Tomo XLVII. Rio de Janeiro: Typographia Nacional, 1884.

2. Morita MC, Haddad AE, Araújo ME. Perfil atual e tendências do cirurgião-dentista brasileiro. Maringá: Dental Press; 2010.

3. Ministério da Educação. Conselho Nacional de Educação. Câmara de Educação Superior. Resolução CNE/CES 3, de 19 de fevereiro de 2002. Institui Diretrizes Curriculares Nacionais do Curso de Graduação em Odontologia. Diário Oficial da União, 4 mar, 2002.

4. Guedes Pinto AC. A história da Odontopediatria no Brasil. São Paulo: Santos; 2014.

5. European Academy of Paediatric Dentistry. A guideline framework for undergraduate education in Paediatric Dentistry. Eur Arch Paediatr. 2010; 10(2):114-9.

6. Cadastro Nacional de Cursos e Instituições de Educação Superior Cadastro e-MEC. [Acesso em 22 fev. 2018]. Disponível em: http://emec.mec.gov.br/.

7. Tanzawa T, Futaki K, Tani C, Hasegawa T, Yamamoto $\mathrm{M}$, Miyazaki $\mathrm{T}$, et al. Introduction of a robot patient into dental education. Eur J Dent Educ. 2012; 16(1):e195-9.

8. Suvinen TI, Messer LB, Franco E. Clinical simulation in teaching preclinical dentistry. Eur J Dent Educ. 1998; 2(1):25-32.

9. American Academy of Pediatric Dentistry. Perinatal and infant oral health care. Pediatr Dent. 2018; 40(6):216-20.

10. American Academy of Pediatric Dentistry. Get it done in year one. [publicação online] 
[Acesso em 8 ago 2019]. Disponível em http://www.mychildrensteeth.org/assets/2/7 /GetItDoneInYearOne.pdf.

11. Pinto VG. Saúde Bucal no Brasil. Rev Saúde Públ. 1983; 17:316-27.

12. Massara MLA, Barbosa PC. Manual de Referência para procedimentos clínicos em odontopediatria. 2 ed. São Paulo: Santos; 2017.

13. Haddad AE, da Cruz DS, Bönecker M.
Odontopediatria ao alcance de todos Práticas Clínicas para os serviços público e privado. São Paulo: Santos; 2020.

\section{Correspondência para:}

Ana Estela Haddad e-mail: aehaddad@usp.br

Av. Professor Lineu Prestes, 2227

Cidade Universitária 05508-000 São Paulo/SP 